\title{
Transient optical properties of highly excited dielectric materials: Apparent birefringence and delayed reflectivity increase
}

\author{
Søren H. Møller $\odot,{ }^{*}$ Sebastian T. Andersen, and Peter Balling $\odot^{\dagger}$ \\ Department of Physics and Astronomy, Aarhus University, 8000 Aarhus C, Denmark
}

(Received 30 June 2020; accepted 2 September 2020; published 1 October 2020)

\begin{abstract}
The ultrafast dynamics of short-pulse-laser-excited fused silica is investigated by measuring polarizationresolved reflectances of a wavelength-tunable probe pulse following a near-infrared pump pulse. These data, which should, in principle, allow the determination of both real and imaginary parts of the time-dependent electric permittivity, turn out to become incompatible with an isotropic optical response after $\sim 200 \mathrm{fs}$. This is reconciled with a uniaxial permittivity tensor, which predicts greatly enhanced absorption of $p$-polarized light, suggesting that an absorption channel is missing in the standard optical models for strongly excited dielectrics. The timescale for the excitation-induced variations in the optical properties exhibits a wavelength dependence that can be explained qualitatively by Pauli blocking in the electronic band structure.
\end{abstract}

DOI: 10.1103/PhysRevResearch.2.043010

\section{INTRODUCTION}

Dielectric materials undergo rapid changes when excited by intense, ultrashort light pulses. The initial modifications result from a drastic rearrangement of electrons between the energy levels (bands) of the material: valence-band (VB) electrons are promoted to the conduction band (CB), typically via strong-field excitation by visible and IR laser pulses [1-4]. The free carriers, i.e., the CB electrons and VB holes, form an electron-hole $(e-h)$ plasma, which is responsible for an increased reflectance (plasma-mirror effect [5]) and absorption in the dielectric material. This absorption plays an important role for energy deposition in the late parts of the laser pulse; it leads to heating of the plasma in a thin layer and opens up for collisional excitation of additional carriers from the VB to CB [3]. After the laser pulse, the carriers thermalize among themselves and, after a few picoseconds (or longer), also with the lattice (atoms). At this stage, if the excitation is strong enough, ablation-removal of atoms from the solid-may be initiated, e.g., if the thermal energy of atoms exceeds their binding energy.

Tracking the changes in material properties with high temporal resolution can be accomplished in pump-probe schemes using ultrashort laser pulses [6-16]. Such experiments provide valuable insight into the transient states of laser-excited materials and can guide theoretical descriptions of the dynamics also when explaining new phenomena, like the recently discovered light amplification in sapphire and

\footnotetext{
*soerenhm@phys.au.dk

†balling@phys.au.dk

Published by the American Physical Society under the terms of the Creative Commons Attribution 4.0 International license. Further distribution of this work must maintain attribution to the author(s) and the published article's title, journal citation, and DOI.
}

fused silica $[17,18]$. The optical properties are governed by the complex-valued dielectric function (relative permittivity, henceforth simply "permittivity") $\varepsilon$, which is therefore an informative quantity to infer, particularly because it can readily be compared between different experimental setups. Through polarization-resolved reflectance measurements, the time-dependent $\varepsilon$ of the excited solid can be determined $[6,7,13,19,20]$.

Here, we report on time- and polarization-resolved reflectance measurements on fused silica $\left(\mathrm{a}-\mathrm{SiO}_{2}\right)$, excited well above the ablation threshold, with a focus on the ultrashort timescale after excitation. We used wavelength-tunable probe pulses with the original goal of experimentally determining the transient permittivity $\varepsilon$. To our surprise, the measured reflectances of $p$ - and $s$-polarized light at all applied wavelengths (480-730 nm) become-and remain-incompatible with an isotropic response of the excited solid $\sim 200 \mathrm{fs}$ after excitation. A uniaxial permittivity tensor correctly describes the reflectance data by producing a greatly enhanced absorption of $p$-polarized light, associated with $\operatorname{Re} \varepsilon \approx 0$. We discuss that the anisotropy may indicate an additional absorption channel for $p$-polarized light that is missing in the standard optical models for fused silica.

\section{EXPERIMENTAL APPROACH}

The experimental setup is a pump-probe scheme, sketched in Fig. 1. The pump and probe pulses derive from a regeneratively amplified Ti:sapphire laser. The pump pulse (of duration $45 \mathrm{fs}$ FWHM) is focused at normal incidence to a spot size (radius at $1 / e^{2}$ ) of $30 \mu \mathrm{m} / 23 \mu \mathrm{m}$ (vertical/horizontal) onto the dielectric target. An optical parametric amplifier produces wavelength-tunable probe pulses (durations of $50 \mathrm{fs}$ or less). The polarization of the probe beam is adjusted to contain almost equal mixtures of $s$ - and $p$-polarized components on the sample, onto which it is focused at an incidence angle $\theta=$ $59.5^{\circ} \pm 1.0^{\circ}$. The reflected probe pulse, which is significantly 


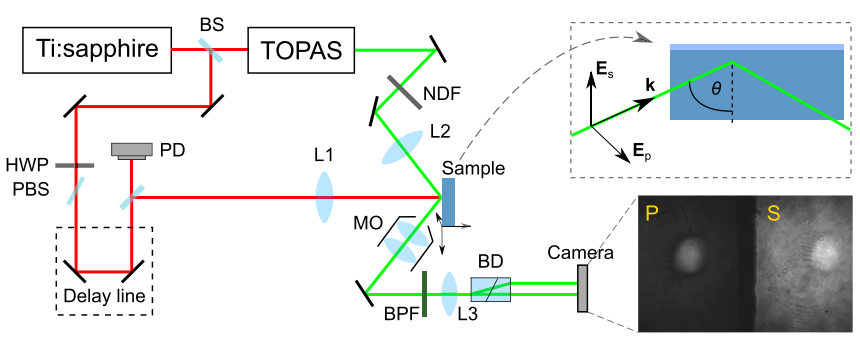

FIG. 1. Pump-probe setup for measuring simultaneously $s$ and $p$ reflectances. The pump and probe beam paths are marked by red and green, respectively. The upper right inset shows the probe beam incident on the sample at an angle $\theta=59.5^{\circ} \pm 1.0^{\circ}$ of mixed $s$ (electric field $\mathbf{E}_{s}$ ) and $p$ (electric field $\mathbf{E}_{p}$ ) polarization. A microscope objective (MO) collects the reflected probe and images it onto a camera, and a beam displacer (BD) separates the reflected $s$ and $p$ components; an example of a resultant image is shown next to the camera (left: $p$, right: $s$ ). HWP: half-wave plate; BS: beam splitter; PBS: thin-film polarizing beam splitter; PD: photodiode, BPF: bandpass filter; NDF: neutral density filter wheel; L1-L3: 100-, 50-, and 200-mm achromatic lenses.

larger than the pump beam on the sample, is imaged via a microscope objective $(20 \times$, numerical aperture $=0.42)$ onto a camera (complementary metal-oxide semiconductor, $10 \mathrm{bit}$ ). A birefringent calcite crystal spatially separates the $s$ - and $p$-polarized components of the probe pulse onto two distinct sections on the camera sensor, allowing simultaneous capture of $s$ - and $p$-polarized images of the pump-excited surface. Polarization filters placed in front of the camera chip ensure that scattered $s$-polarized light is blocked on the $p$-polarized section of the camera and vice versa. An electromechanical shutter is placed in the pump beam, which allows us to selectively block the pump and thus capture probe images of the sample surface before excitation with the pump. The absolute reflectances of the pump-probe images are obtained by normalizing against probe-only images and the known reflectances of the pristine sample. The data-analysis procedure is briefly described in Appendix A.

\section{EXPERIMENTAL RESULTS}

Figure 2 shows a measurement acquired on $\mathrm{a}-\mathrm{SiO}_{2}$ with 700-nm probe pulses. The average pump-pulse energy was $62 \mu \mathrm{J}$, corresponding to a peak fluence of about $5.7 \mathrm{~J} \mathrm{~cm}^{-2}$ (peak intensity $\sim 100 \mathrm{TW} \mathrm{cm}^{-2}$ ), which is about 2 times the ablation threshold fluence $\left(\sim 2.9 \mathrm{~J} \mathrm{~cm}^{-2}\right)$. Figures 2 (a) and 2(b) contain selected images of the $p$ - and $s$-polarized reflectances $\left(R_{p}\right.$ and $\left.R_{s}\right)$, respectively, at different delay times. The emerging bright central spot reflects the large free-carrier density created by the pump pulse, viewed under an angle of $60^{\circ}$. Figures 2(c) and 2(d) show the detailed time evolution of $R_{p}$ and $R_{s}$ along a fixed vertical slice passing through the pump-spot centers in Figs. 2(a) and 2(b), while Fig. 2(e) shows the reflectances in the center of the pump-excited area. The zero delay $(t=0)$ was inferred from reflectance measurements of the Kerr effect on a different sample (see Appendix C). Because a sample change is involved in estimating $t=0$ for our a-SiO $\mathrm{Si}_{2}$ measurements, we expect that the zero-delay value is uncertain by about $100 \mathrm{fs}$.

Two prominent features are observed in Fig. 2(e): (i) Both $R_{s}$ and $R_{p}$ increase until $\sim 200$ fs after excitation, which is longer than the pump-probe overlap of 70 fs FWHM (Appendix $\mathrm{C}$ ), and (ii) $R_{p}$ exhibits a subsequent decrease between 200 and $300 \mathrm{fs}$. We quantify these two aspects by parametrizing the transient reflectance in the central part of the pump beam by error functions, which gives rise times $\tau_{k}(k=p, s)$ and the "final" values $R_{k, \infty}$ (i.e., for delays $t \gtrsim 400 \mathrm{fs}$ ):

$$
\begin{aligned}
& R_{s}(t) \sim R_{s, 0}+\left(R_{s, \infty}-R_{s, 0}\right) F_{s}(t), \\
& R_{p}(t) \sim R_{p, 0}+\left(R_{p, \infty}-R_{p, 0}\right) F_{p}(t)+\Delta R_{p} G_{p}(t),
\end{aligned}
$$

with $\quad F_{k}(t)=\operatorname{erf}\left[\sqrt{4 \ln 2}\left(t-t_{0, k}\right) / \tau_{k}\right] / 2$ and $G_{p}(t)=$ $\exp \left[-4 \ln 2\left(t-t_{0, p}^{2}\right) / \tau_{p}^{2}\right] . R_{k, 0}$ are the reflectivities of fused silica. The expression for $R_{p}$ includes also a Gaussian term $G_{p}$ that accounts for the subsequent reflectance drop. The successful reproduction of the reflectance data across all the applied wavelengths with Eq. (1) is shown in Appendix B.

The values of $R_{k, \infty}$ and $\tau_{k}$ are shown as a function of probe frequency (wavelength) in Fig. 3, where all values are obtained at the center of the pump pulse. $R_{s, \infty}$ generally
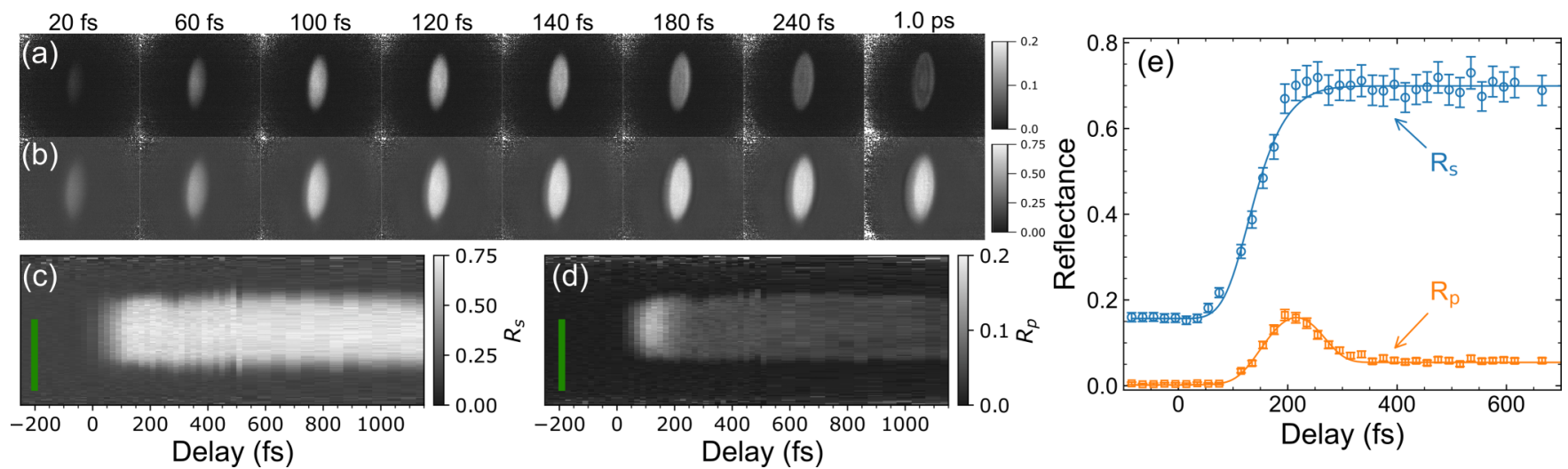

FIG. 2. Reflectance at $700 \mathrm{~nm}$ of a-SiO 2 after pump excitation. (a) and (b) Selected images at different time delays of (a) $R_{p}$ and (b) $R_{s}$. (c) and (d) Reflectance time series along a vertical slice passing through the center of the pump spots in (a) and (b). The green bars indicate $30 \mu \mathrm{m}$. (e) Time evolution of $R_{s}$ (blue circles) and $R_{p}$ (orange squares) in the center of the pump spot. Solid lines represents fits of Eqs. (3) and (5) to the data. The zero delay is uncertain by about $100 \mathrm{fs}$. 


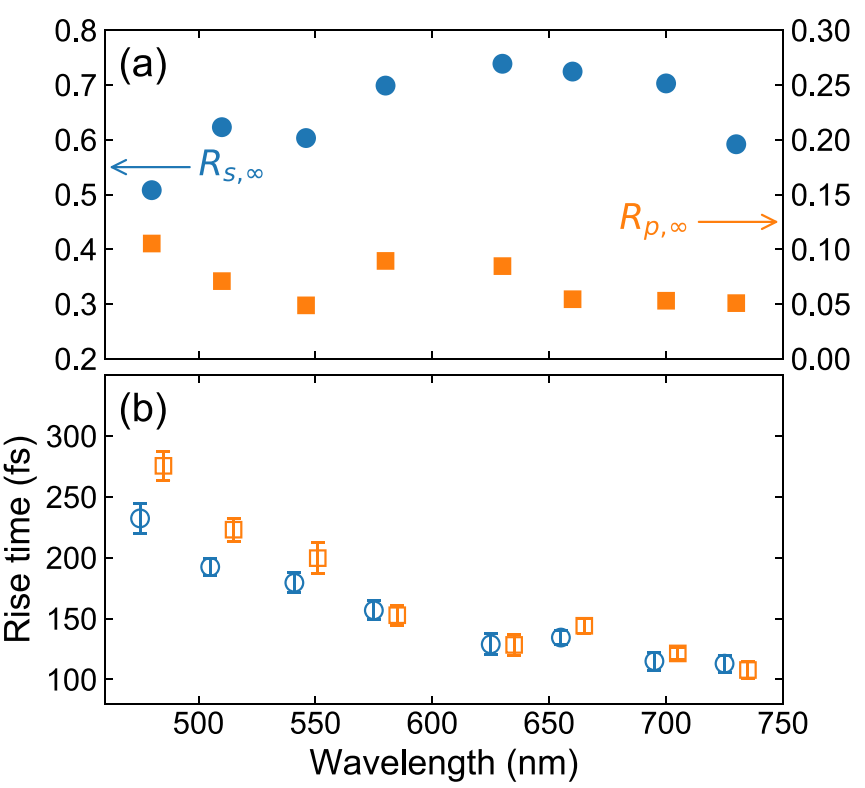

FIG. 3. Values of (a) $R_{k, \infty}$ and (b) $\tau_{k, \infty}$ for $k=s$ (blue circles) and $k=p$ (orange squares), estimated from the reflectance data at different probe wavelengths. The relative positions of $\tau_{s}$ and $\tau_{p}$ in (b) have been shifted slightly along the horizontal axis to avoid overlapping data.

increases with wavelength, which is expected from an $e-h$ plasma in the excited solid, where CB electrons and VB holes screen and reflect more effectively long-wavelength light. However, $R_{p, \infty}$ remains low and is less sensitive to the wavelength; this surprising feature already suggests that a simple Drude model for $\varepsilon$ of the $e$ - $h$ plasma cannot account for the data across all wavelengths. Another interesting observation is the systematic increase in rise times for shorter probe wavelengths. In fact, the smallest values of $\tau_{k}$ at $730 \mathrm{~nm}$ are still longer than the expected cross correlation between the pump and probe of $70 \mathrm{fs}$. Relatively slow rise times of the reflectance under ultrafast laser excitation were reported before in [12]. An explanation in terms of gradual band filling and Pauli blocking is given in Sec. V A.

\section{TIME-DEPENDENT $\varepsilon$}

From the time-dependent $p$ - and $s$-polarized reflectances $\left(R_{p}\right.$ and $\left.R_{s}\right)$ it is, as mentioned previously, possible to infer the complex permittivity $\varepsilon$ from Fresnel's equations for $R_{S}$ and $R_{p}[6,7,13,19,20]$. However, excitation by a strong laser pulse creates a quite inhomogeneous excitation in depth of the material (the variation in the transverse directions can be neglected in comparison), so Fresnel's equations are not strictly valid. When this complication is neglected (as is often the case), the inferred $\varepsilon$ represents rather a so-called pseudo- $\varepsilon$ [21]. To improve this, we assume that the laser-induced change in $\varepsilon$ inside the material decreases exponentially away from the surface (located at $z=0$ ) with a range $L$, i.e.,

$$
\varepsilon(z, \omega)=\varepsilon_{\mathrm{SiO}_{2}}(\omega)+\Delta \varepsilon \mathrm{e}^{-z / L} \quad(z \geqslant 0),
$$

where $\varepsilon_{\mathrm{SiO}_{2}}(\omega)$ is the unperturbed dielectric function of fused silica [22] and $\Delta \varepsilon$ is the change in $\varepsilon$ due to the pump-

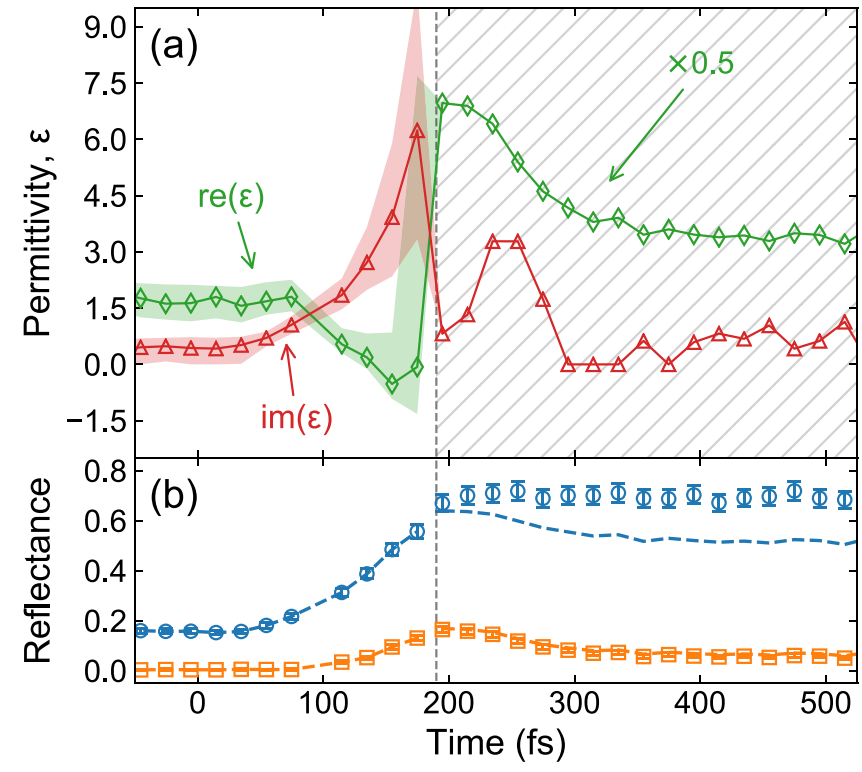

FIG. 4. Determination of the time-dependent permittivity $\varepsilon$ at the surface from $R_{p}$ and $R_{s}$ at $700 \mathrm{~nm}$ [same data as in Fig. 2(e)]. (a) Real (green diamonds) and imaginary (red triangles) parts of the most likely values of $\varepsilon$ according to the data. The shaded ribbons show $1 \sigma$ credible intervals where $\varepsilon$ correctly reproduce the reflectances. (b) $R_{s}$ and $R_{p}$ (blue circles and orange squares) at different time delays. The solid lines are the reflectance calculated from $\varepsilon$ in (a). When $t>200$ fs [hatched section in (a)], no $\varepsilon$ can reproduce the observed data.

pulse excitation. With a depth-dependent $\varepsilon$, the reflectances of $s$ - and $p$-polarized light, incident at $59.5^{\circ}$, are obtained by numerically integrating the Helmholtz equations (see Appendix D).

In the present case, it turns out that we cannot reproduce the experimental data with any $\Delta \varepsilon$ once $R_{p}$ decreases after its initial rise. This is exemplified in Fig. 4 for $700 \mathrm{~nm}$ [same data as in Fig. 2(e)], where $\varepsilon$ obtained from the measurements is successful only for $t<200 \mathrm{fs}$. Initially, we observe that the real and imaginary parts of $\varepsilon$ ( $\operatorname{Re} \varepsilon$ and $\operatorname{Im} \varepsilon$ ) decrease and increase, respectively; this matches the expected optical response of an $e-h$ plasma. The values of $\varepsilon$ obtained for $t>200 \mathrm{fs}$ represent the "best" fit to the data (minimal mean-square deviation between calculated and observed reflectances) but completely fail to reproduce the data as clarified in Fig. 4(b). Adjusting the range $L$ over which $\varepsilon$ decreases in depth does not change these conclusions.

\section{A. Anisotropic model}

Since an isotropic (but depth-dependent) permittivity fails to simultaneously describe $s$ reflectance $R_{s}$ and $p$ reflectance $R_{p}$ when the latter starts to decrease, as a straightforward extension, we introduce an anisotropic permittivity tensor where the electric response in the $z$ direction $\varepsilon_{z z}$ is different from the in-plane electric response in the $x$ and $y$ directions; $\varepsilon_{z z} \neq \varepsilon_{x x}=\varepsilon_{y y}$. Off-diagonal terms like $\varepsilon_{x z}$ are set to zero. We discuss these assumptions after we have examined how well this description can reproduce the transient reflectance data. 
As in the isotropic case, the goal is to obtain the timedependent permittivity tensor from pairs of $\left(R_{s}, R_{p}\right)$. However, direct "inversion" is challenging because we, at each pumpprobe delay, have four unknowns (real and imaginary parts of $\varepsilon_{x x}$ and $\varepsilon_{z z}$ ) and only two observations with an experimental uncertainty. Instead, we hypothesize a suitable time-dependent evolution of the permittivity containing only a few free parameters. It is usually found that a Drude model adequately describes the optical properties of laserexcited band-gap materials. Accordingly, the in-plane timeand position-dependent permittivity at probe frequency $\omega$ is described as

$$
\varepsilon_{x x}(z, t)=\varepsilon_{\mathrm{SiO}_{2}}+\left[\left(1-\varepsilon_{\mathrm{SiO}_{2}}\right) \frac{n(z, t)}{n_{\mathrm{val}}}-\frac{e^{2} n(z, t)}{\varepsilon_{0} m \omega(\omega+i \Gamma)}\right],
$$

with the free-carrier density

$$
n(z, t)=\frac{n_{0}}{2} \mathrm{e}^{-z / L}\left[1+\operatorname{erf}\left(\frac{\sqrt{4 \ln 2} t}{\tau_{n}}\right)\right] .
$$

$\Gamma$ is a phenomenological Drude scattering rate (see, e.g., [23] for a discussion), $m=0.43 m_{\mathrm{e}}$ is the reduced effective $e-h$ mass $\left(m_{\mathrm{e}}=9.11 \times 10^{-31} \mathrm{~kg}\right)$, and $n_{\text {val }}=3.2 \times 10^{23} \mathrm{~cm}^{-3}$ is the VB electron density [16]. We are here assuming that the electron and hole densities and scattering rates are equal so that their collective response is described in terms of the effective mass. The term containing $n / n_{\text {val }}$ accounts for the change in optical properties due to depletion of the VB in fused silica. The electron density has an error-function growth with rise time $\tau_{n}$.

The permittivity in the $z$ direction is expressed as $\varepsilon_{z z}=$ $\varepsilon_{x x}+\chi$, where

$$
\chi(z, t)=\frac{\chi_{0}}{2} \mathrm{e}^{-z / L}\left[1+\operatorname{erf}\left(\frac{\sqrt{4 \ln 2}\left(t-t_{0}\right)}{\tau_{\chi}}\right)\right] .
$$

$\chi_{0}$ is treated as a complex-valued free fitting parameter that describes the amount of anisotropy; when it is zero, the permittivity tensor is isotropic. The time dependence of $\chi$ is similar to that of the electron density, but with a potentially different rise time $\tau_{\chi}$ and delay $t_{0} \geqslant 0$.

\section{B. Results}

The time-resolved anisotropic permittivity tensor of the excited fused silica is obtained by first fitting Eq. (3) to the parameterized $s$-polarized data [i.e., Eq. (1)] for a fixed value of $L$, allowing four parameters to vary: $n_{0}, \Gamma, \tau_{n}$, and a temporal offset which sets the time at which $R_{s}$ has increased to half of its value (this is somewhat later than the delay zero, where the pump and probe simultaneously strike the sample surface). Equation (5) is subsequently fitted to the parameterized $p$-polarized data using four free parameters: the real and imaginary parts of $\chi_{0}, \tau_{\chi}$, and $t_{0}$. To avoid overconstraining the optimization of the parameters, data sets at different probe wavelengths are fit independently. The entire procedure is repeated for a range of values of $L$, from which we select the "optimal" value (largest likelihood): $L=40 \pm 2 \mathrm{~nm}$. This value appears rather small, but it is needed to suppress an otherwise pronounced dip in $R_{S}$, not seen in the data, when the real part of $\varepsilon$ passes through zero from positive values.
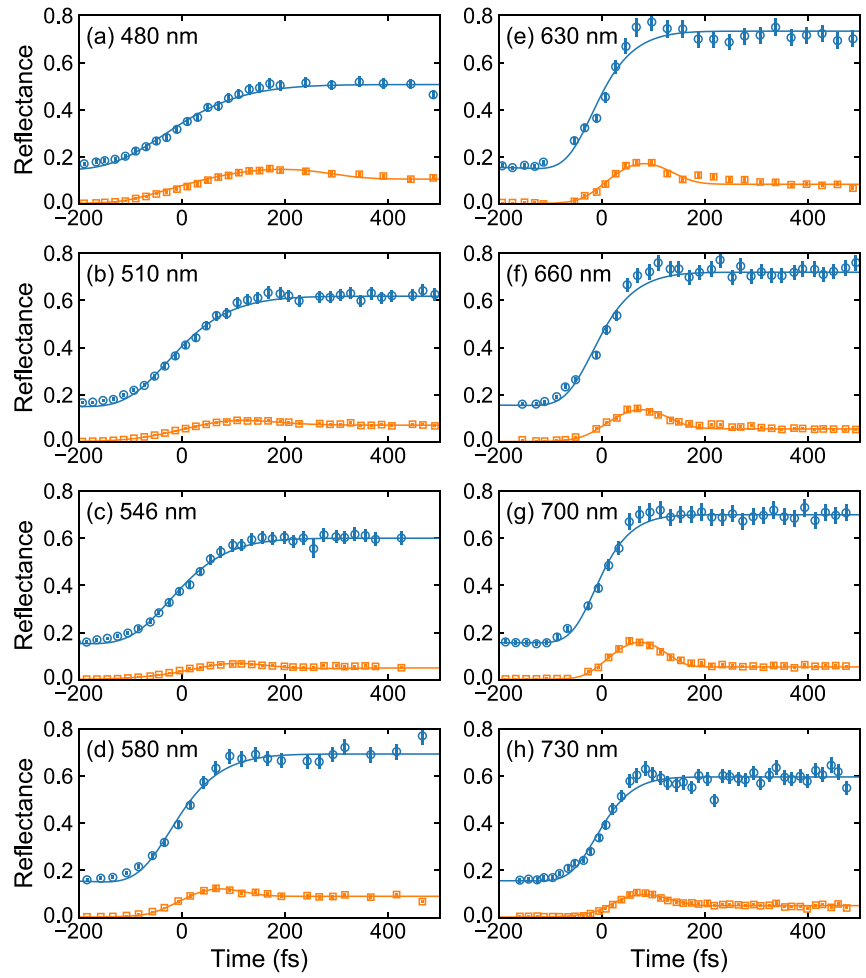

FIG. 5. (a)-(h) Time-dependent $s$ - and $p$-reflectance data (blue circles and orange squares, respectively) at different probe wavelengths. The solid lines are the corresponding predictions by the anisotropic permittivity model, defined by Eqs. (3)-(5). The time zero is defined by Eq. (4).

As shown in Fig. 5, the anisotropic permittivity tensor successfully reproduces the $R_{s}$ and $R_{p}$ data for the different applied probe wavelengths. Since the $s$ polarization probes only $\varepsilon_{x x}$ or $\varepsilon_{y y}$, we can conclude that the Drude model adequately describes $R_{s}$, in agreement with previous references $[11,14,16]$. The final "steady" values of $\varepsilon_{x x}$ (i.e., for $t>$ $400 \mathrm{fs}$ ), displayed in Fig. 6(a), show a typical Drude-like dispersion where the real and imaginary parts of the permittivity increase with wavelength. The fitted Drude parameters agree well across the entire range of probe wavelengths employed here (see Fig. 10 in Appendix E); the averaged values are supplied in Table I and (apart from $L$, which is usually considered infinite) agree well with previous experiments employing strong ultrashort laser pulses $[6,10,11,14,16]$. The full timedependent evolution of $\varepsilon_{x x}$ at $700 \mathrm{~nm}$ is shown by the solid lines in Fig. 6(c).

The final values of $\varepsilon_{z z}$, shown in Fig. 6(b), are intriguing: optimal values of $\chi_{0}$ push the real part of $\varepsilon_{z z}$ towards zero at the sample surface for most of the applied probe wavelengths. Apparently, near the surface, the electric response in $z$ resembles that of an epsilon-near-zero material which generally enhances light absorption [24,25], for instance, via excitation of plasmons or plasmon polaritons in the material $[26,27]$. We discuss these aspects in the next section.

\section{DISCUSSION}

Our pump-probe measurements on fused silica show two surprising features: (i) wavelength-dependent rise times of the 


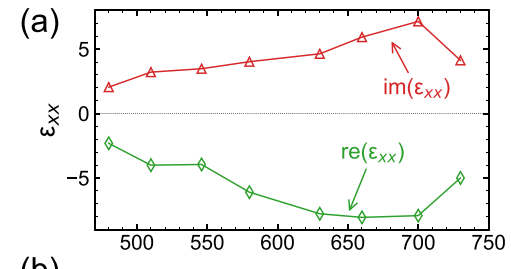

(b)
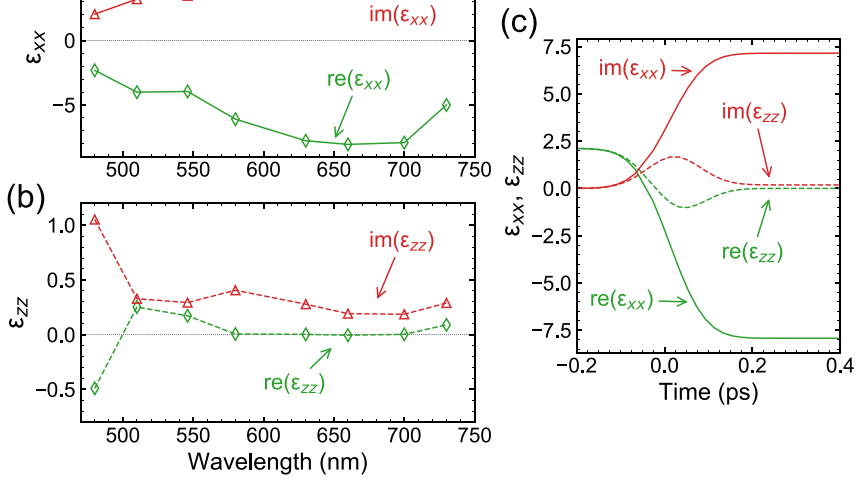

FIG. 6. Final "steady-state" components (0.4-1 ps after the pump) of the permittivity tensor; (a) and (b) real (green diamonds) and imaginary (red triangles) parts of $\varepsilon_{x x}$ and $\varepsilon_{z z}$ at different probe wavelengths. (c) Time evolution of $\varepsilon_{x x}$ (solid lines) and $\varepsilon_{z z}$ (dashed lines) at $700 \mathrm{~nm}$. These permittivities produce the solid lines of $R_{S}$ and $R_{p}$ shown in Fig. 2(e) and in Fig. 5 at $700 \mathrm{~nm}$.

reflectance with shorter wavelengths exhibiting a slower response (Fig. 3) and (ii) an ultrafast drop in $R_{p}$ after excitation, which could be reconciled with a uniaxial permittivity tensor. We discuss each of these aspects in turn.

\section{A. Slow rise times}

What is the reason for the strong wavelength-dependent rise times observed in both $R_{s}$ and $R_{p}$ (see Fig. 3 or $\tau_{n}$ in Fig. 10 of Appendix E) that, in all cases, are longer than the $\sim 70$ fs interaction time (FWHM) of the pump and probe pulses?

A slow increase in reflectance, which continues after the pump pulse is over, was seen in previous investigations on strongly excited dielectrics $[11,12]$. This behavior is not easy to explain if the dynamics is governed primarily by the growth of the $e-h$ density inside the dielectric target, where the fastest reflectance increase should happen during the pump pulse. Collisional excitation, where energetic free carriers excite additional valence-band electrons across the band gap $[2,3]$, could potentially result in a delayed reflectance increase if the collision rate is low. However, theoretical modeling of the nonequilibrium dynamics of laser-excited dielectrics

TABLE I. Parameters appearing in the permittivity tensor [Eqs. (3) and (5)]: the top and bottom rows show, respectively, fixed and fitted values, with the latter summarized as mean \pm standard deviation based on the results from all the applied probe wavelengths.

\begin{tabular}{lc}
\hline \hline Parameter & Value \\
\hline$m^{\mathrm{a}}$ & $3.92 \times 10^{-31} \mathrm{~kg}$ \\
$n_{\text {val }}{ }^{\mathrm{a}}$ & $3.2 \times 10^{23} \mathrm{~cm}^{-3}$ \\
$n_{0}$ & $1.32 \pm 0.09 \times 10^{22} \mathrm{~cm}^{-3}$ \\
$\Gamma$ & $1.73 \pm 0.09 \times 10^{15} \mathrm{~s}^{-1}$ \\
$L$ & $40 \pm 2 \mathrm{~nm}$ \\
$t_{0}$ & $70 \pm 40 \mathrm{fs}$ \\
\hline \hline
\end{tabular}

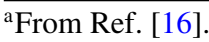

shows that collisions happen on a few-femtosecond timescale $[28,29]$ - much too fast to explain the slow rise times observed here. Furthermore, collisional excitation alone cannot explain the slower optical response for shorter-wavelength probe pulses.

We propose that the slow wavelength-dependent rise times are evidence of band filling and Pauli blocking. To simplify the discussion, we consider just the $s$-polarized reflectance, which is adequately described by the time-dependent Drude model in Eq. (3). Immediately after excitation, we expect that electrons (and holes) occupy a wide range of energy levels in the CB (VB). Through inter- and intraband scattering, the carriers relax to the bottom (top) of the CB (VB). At this stage, $n, \Gamma$, and $m$ are well-defined quantities. However, the large electron densities $\left(10^{22} \mathrm{~cm}^{-3}\right)$ imply that the $\mathrm{CB}$ is degenerate. Because of Pauli blocking, photons of energy $\hbar \omega$ are allowed to interact only with $\mathrm{CB}$ electrons lying at most $\hbar \omega$ below the qausi-Fermi level (similarly for holes). The effective free-carrier density entering the Drude model should therefore have a slight frequency dependence, where larger effective densities are expected for larger frequencies. As a consequence, the reflectance saturates faster for longwavelength probe pulses, while the electrons gradually relax to the bottom of the CB. Of course, the details will depend strongly on the material band structure, but qualitatively, we expect that the saturation of transient reflectance is delayed for low-wavelength probe pulses, in agreement with Fig. 3.

\section{B. Ultrafast drop in $R_{p}$}

From the $R_{s}$ and $R_{p}$ measurements with the applied probe wavelengths between 480 and $730 \mathrm{~nm}$, we see consistently a drop in $R_{p}$ after a few 100 fs (Fig. 5), although the effect is less pronounced for shorter wavelengths. To the best of our knowledge, this effect-the ultrafast drop in $R_{p}$-has been reported only a few times before $[9,15]$. These studies, like ours, used a geometry in which the probe pulse is incident at large angles $\left(46^{\circ}\right.$ and $55^{\circ}$, respectively), whereas most other studies have used near-normal incident probes.

References [9,15] suggest that the low p-polarized reflectance is due to resonance absorption, a process whereby the incident light pulse creates a self-sustained charge-density wave (a "plasmon"). Such an excitation is possible only when the real part of the electric permittivity $\operatorname{Re} \varepsilon$ goes through zero along a density gradient [30]. In our case, the gradient lies along $z$ (along the pump-beam propagation), so plasmon excitation is possible only with $p$-polarized light. However, this explanation based on resonance absorption is not sufficient in itself to describe our results; resonance absorption is already captured in our numerical solutions to the Helmholtz equations, and values of the vanishing real part of an isotropic permittivity close to the surface cannot simultaneously explain the low $p$ reflectance and the high $s$ reflectance. (According to the Drude model that fits our $R_{s}$ data, $\operatorname{Re} \varepsilon$ passes through zero deeper within the solid, but at this point most of the probe pulse has been reflected, producing an $R_{p}$ that is larger than what we measure.)

To account for both the high $R_{s}$ and low $R_{p}$, we introduced birefringence via Eq. (5) in $\varepsilon_{z z}$, and we found that $\operatorname{Re} \varepsilon_{z z} \approx 0$ near the surface gave the best description of $R_{p}$ at 


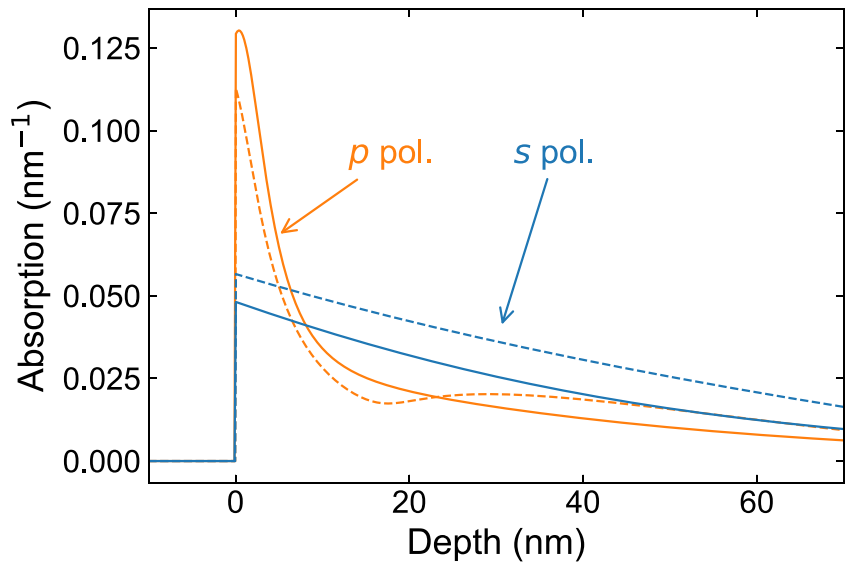

FIG. 7. Depth-dependent absorption coefficients for $p$-polarized (orange) and $s$-polarized (blue) light according to the anisotropic permittivity model [Eqs. (3)-(5)] for a probe pulse at $700 \mathrm{~nm}$ incident at $60^{\circ}$. Near the surface, $\operatorname{Re} \varepsilon_{z z} \approx 0$, which results in a high absorption of $p$-polarized light. The dashed lines are the corresponding estimates using the local expression for the absorption coefficient.

late time delays. If we look at the absorption profile according to our model, we indeed observe an increased absorption of $p$-polarized light near the surface (see Fig. 7). As explained in Ref. [24], a near-zero permittivity implies a vanishingly small group velocity of the pulse, thereby considerably increasing the light-matter interaction time and hence the losses. This is the reason that, in comparison, the $s$-polarized probe pulse suffers much less absorption in our calculations even though the imaginary part of $\varepsilon_{x x}, \operatorname{Im} \varepsilon_{x x}$ (and $\varepsilon_{y y}$ ), greatly exceeds $\operatorname{Im} \varepsilon_{z z}$ [see Fig. 6(a)]. The reduced group velocity and increased absorption of the $p$-polarized probe pulse may be associated with a plasmon or a plasmon polariton $[24,26]$.

The need for an anisotropic optical response with $\operatorname{Re} \varepsilon_{z z} \approx$ 0 is thus indicative of an additional absorption channel for $p$ polarized light that is not captured by an isotropic permittivity in the Helmholtz equation. The unusually flat dispersion of $\varepsilon_{z z}$ in Fig. 6 with a real part close to zero (at least above $550 \mathrm{~nm}$ where the ultrafast drop in $R_{p}$ is more pronounced) seems to support this since it is hard to imagine that a solid could respond in this way over a large bandwidth. It is thus likely that $\varepsilon_{z z}$ serves to represent the optical response corresponding to an additional absorption channel that is not captured in the standard optical models for strongly excited solids.

As an aside, we note that the standard expression for the absorption coefficient of an excited (lossy) dielectric expressed by the local absorption coefficient $\alpha_{k}=2 \kappa_{k} \omega / c$ ( $k=s, p$; dashed lines in Fig. 7) [3,31] agrees quite well with our calculations based on solving the Helmholtz equation (solid lines) with $\kappa_{s}=\operatorname{Im} \sqrt{\varepsilon_{x x}-\sin ^{2} \theta}$ and $\kappa_{p}=$ $\operatorname{Im} \sqrt{\varepsilon_{x x}-\varepsilon_{x x} \sin ^{2} \theta / \varepsilon_{z z}}$ [32]. This is somewhat surprising since the concept of a local extinction coefficient is based on spatial variations of the permittivity happening on length scales that are larger than the probe wavelengths [33]. This is clearly not fulfilled in the present case; the ability to use the local extinction coefficient is, however, very convenient when modeling excitation of and propagation of light in dielectric materials [3].
Finally, let us remark that strong-field-induced anisotropy was recently predicted several times in time-dependent density-functional-theory (TDDFT) simulations [34-36]. However, this type of anisotropy is quite different from the (apparent) anisotropy reported here; in TDDFT, anisotropy emerges because a linearly polarized pump field produces an inhomogeneous distribution of excited carriers in reciprocal space, an effect which is expected to diminish after 1-100 fs associated with the carrier-momentum relaxation time in a strongly excited solid [37].

\section{Self-trapped excitons and Coulomb explosion?}

Let us discuss briefly the role of two effects, self-trapped excitons and Coulomb explosion, that have been postponed so far.

Self-trapped excitons (STEs) are known to form quickly (well before $1 \mathrm{ps}$ ) after laser excitation of fused silica [8,38]; they strongly influence the optical properties by increasing the real part of the permittivity. These optical effects due to STEs can easily be implemented in Eq. (3) as described in [16]. However, we are justified in not considering this effect since STE formation is suppressed near the surface because the strong Coulomb potential at large free-carrier densities $\left(\gtrsim 10^{22} \mathrm{~cm}^{-3}\right.$ ) effectively screens the $e-h$ interaction [16]. As a result, the optical signature of STEs is extremely weak in reflection measurements which probe primarily the surface, in contrast to transmission measurements, where signatures of STEs are frequently observed.

Another effect to consider is Coulomb explosion, where a thin surface layer disintegrates due to mutual repulsion between positive ions that were stripped of electrons through photoemission, stimulated by the laser pulse $[39,40]$. Assuming that Coulomb explosion happens, a thin plasma cloud may be formed in front of the surface; it is possible to tune this layer to reproduce a drop in $R_{p}$ without noticeable effects in $R_{S}$ due to interference between the plasma layer and the solid (our simulations have confirmed this). However, the optical signature, being fairly sensitive to small variations in the cloud, cannot produce a consistently low $R_{p}$ for delays over a few hundred femtoseconds because the velocity of the ejected electrons is initially too large. Besides, a vast number of electrons (densities around $10^{21} \mathrm{~cm}^{-3}$ or more) must be emitted for the plasma cloud to have a noticeable optical effect in our experiment, which would initially produce a huge electrostatic potential, comparable in magnitude to the energy pumped into the system. Thus, it is highly unlikely that a dense plasma cloud can explain these effects (we cannot exclude a dilute plasma cloud as implied by Coulomb explosion, but its optical signature is then insignificant).

\section{SUMMARY}

By measuring polarization-resolved reflectances of fused silica, excited by an ultrashort pump pulse above the ablation threshold, using a wavelength-tunable probe pulse, we found that any isotropic optical response fails to simultaneously describe the observed $s$ and $p$ reflectances $\sim 200 \mathrm{fs}$ after excitation. We argued that an additional absorption channel is necessary to explain the low reflectance of $p$-polarized light. 
In addition, we observed a somewhat slow (on the 100-fs timescale) evolution of the optical properties with a strong wavelength dependence, which can be explained by Pauli blocking and band-filling effects.

\section{ACKNOWLEDGMENTS}

We thank L. Haahr-Lillevang for his contributions to an early version of this experiment and T. Winkler and B. Julsgaard for helpful comments and discussion. Innovation Fund Denmark is acknowledged for funding via the SunTune project.

\section{APPENDIX A: DATA ANALYSIS}

To estimate the change in surface reflectance induced by the pump at a specific pump-probe delay, two images are captured: first, a reference image $\mathbf{r}=\left\{r_{i}\right\}$ of the surface without the pump and then an image with the pump and probe, $\mathbf{d}=$ $\left\{d_{i}\right\}$. The difference between $\mathbf{d}$ and $\mathbf{r}$ gives the signal change $\mathbf{x}=\left\{x_{i}\right\}$ due to the pump excitation, which is proportional to the reflectance change.

A single picture captured on the camera actually contains two images of the surface acquired with the $p$ and $s$ polarizations of the probe, respectively (see Fig. 1). We consider these two images to be separate; that is, the $p$-polarized image is $\mathbf{d}^{(p)}=\left\{d_{i}^{(p)}\right\}$, and the $s$-polarized image is $\mathbf{d}^{(s)}=\left\{d_{i}^{(s)}\right\}$. We leave out the superscripts indicating the polarization when the formulas apply equally well to both cases.

Because of pulse-to-pulse energy fluctuations of the probe (fluctuations are much more pronounced for the probe because it is generated through nonlinear processes of the pump), we model the data as

$$
d_{i}=a\left(r_{i}+x_{i}\right),
$$

where $a$ is the ratio of the energies of the two subsequent probe pulses. The regions outside the pump-beam-affected zone, where $x_{i}=0$, are used to estimate the probe-energy ratio $a$. Once $a$ is known, $x_{i}$ can be estimated everywhere from Eq. (A1).

To get absolute reflectances of the $p$ and $s$ components during pump-pulse excitation, we must know the reflectances of the pristine sample, here denoted as $R_{p, 0}$ and $R_{s, 0}$. They are estimated from the Fresnel equations given the known angle of incidence $\theta \approx 60^{\circ}$ and known refractive index of the sample at the probe wavelength [22]. For a particular pixel (suppressing the $i$ indices in the following), the associated reflectance change is

$$
\Delta R_{k}=\frac{x^{(k)}}{r^{(k)}} R_{0, k}, \quad k=s, p .
$$

Because the $60^{\circ}$ incidence angle is close to the Brewster angle of fused silica, $r^{(p)} \approx 0$, which makes Eq. (A2) useless for estimating $\Delta R_{p}$. Instead, we use the reference image captured on the $s$ side of the camera, properly translated and scaled, so that according to Eq. (A2),

$$
\Delta R_{p}=\gamma \frac{x^{(p)}}{x^{(s)}} \Delta R_{s}
$$
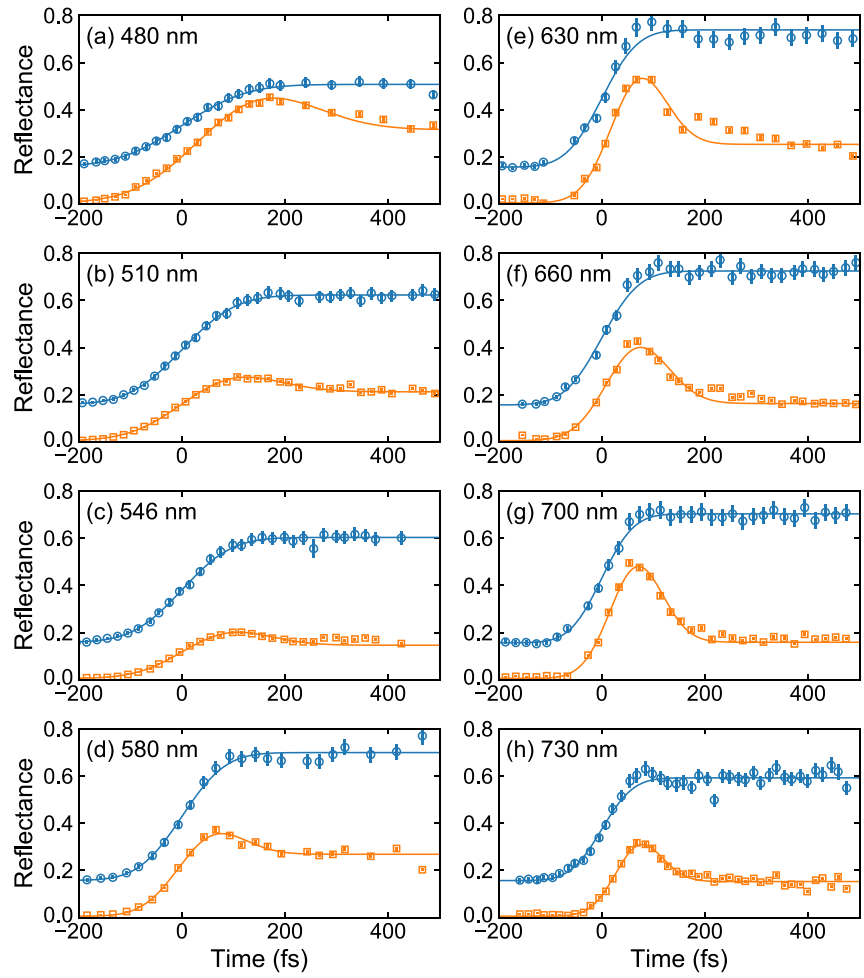

FIG. 8. (a)-(h) Time-resolved $R_{s}$ (blue circles) and $R_{p}$ (orange squares) data in the center of the pump beam spot at different probe wavelengths. For clarity, $R_{p}$ has been scaled up by 3 , and the data have been offset in time for ease of comparison. The solid lines are the parametrizations via Eq. (1).

We have introduced the factor $\gamma$ to correct for the relative pulse energies in the $s$ and $p$ components of the probe. $\gamma(\approx 1)$ is acquired by calibrating the camera using a highly reflective silver mirror.

The absolute reflectances reported in the main text are obtained as $R_{k}=R_{k, 0}+\Delta R_{k}$. The data analysis procedure is carried out automatically using Bayesian probability theory as described later in Appendix F.

\section{APPENDIX B: PARAMETRIZATION BY ERROR FUNCTIONS}

The time-resolved reflectance measurements, displayed, for example, in Figs. 2(e) and 5, can be parametrized quite well by Eq. (1); this is demonstrated in Fig. 8, which compares the experimental values (circles and squares) with the parametrized curves (solid lines). Equation (1) provides the model-free time constants in Fig. 3.

\section{APPENDIX C: TEMPORAL PUMP-PROBE OVERLAP}

The temporal overlap between the pump and probe was obtained from reflectance measurements on homemade samples consisting of $\mathrm{GaN}$ nanowires grown on top of silicon. An example of such a measurement at $700 \mathrm{~nm}$ is shown in Fig. 9 . The strong pump field changes the instantaneous refractive index (Kerr effect), modifying the reflectance of the probe when the two pulses overlap in time. The point where the pump-probe overlap is maximized $(t=0)$ is marked by the 


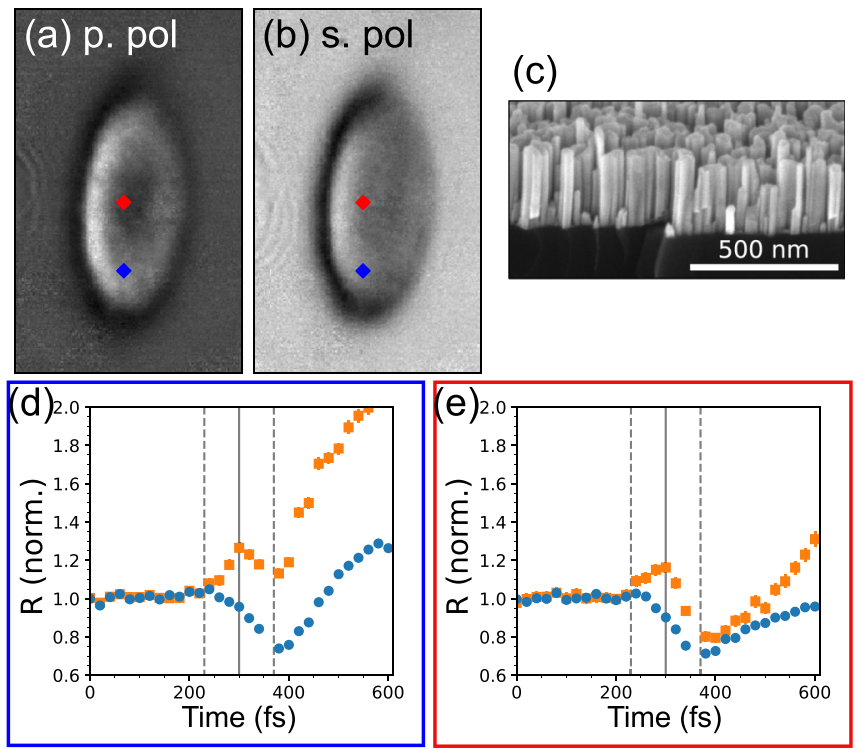

FIG. 9. Time-resolved reflectance measurements of $\mathrm{GaN}$ nanowires at $700 \mathrm{~nm}$. (a) and (b) Images of the (a) $p$ and (b) $s$ components of the probe at a certain time delay. (d) and (e) The time evolution of the two regions marked by red and blue squares, respectively. Normalized values of the $p$ and $s$ reflectances are represented, respectively, by blue circles and orange squares. The vertical dashed lines indicate the region of the presumed temporal overlap of the pump and probe pulses. (c) A cross-sectional SEM image of the $\mathrm{GaN}$ nanowires.

solid vertical line. The dashed lines put the FWHM of the pump-probe overlap close to $70 \mathrm{fs}$.

\section{APPENDIX D: REFLECTANCE CALCULATIONS}

The permittivity tensor $\left(\varepsilon_{x x}=\varepsilon_{y y}, \varepsilon_{x y}=\varepsilon_{y z}=\varepsilon_{z x}=0\right)$ considered in the main text is $z$ dependent (the sample surface is located at $z=0$ ), so the Helmholtz equations must be solved numerically by integration or by use of the transfermatrix method (TTM) [41]. We employ the former approach.

For an $s$-polarized probe at frequency $\omega$ incident on the sample at the angle $\theta$ with respect to the surface normal ( $x y$ plane), the electric field is polarized in the $y$ direction; the Helmholtz equation for the electric field reads

$$
E_{y}^{\prime \prime}+\frac{\omega^{2}}{c^{2}}\left(\varepsilon_{y y}-\sin ^{2} \theta\right) E_{y}=0 .
$$

The prime denotes differentiation with respect to $z$.

For the $p$-polarized probe, the equation becomes more complicated because the field has components in both $x$ and $z$, and it is simpler to consider the magnetic field, which is polarized in the $y$ direction. The equation for the magnetic field is

$$
B_{y}^{\prime \prime}-\frac{\varepsilon_{x x}^{\prime}}{\varepsilon_{x x}^{2}} B_{y}^{\prime}+\frac{\omega^{2}}{c^{2}}\left(\varepsilon_{x x}-\frac{\varepsilon_{x x}}{\varepsilon_{z z}} \sin ^{2} \theta\right) B_{y}=0 .
$$

The two uncoupled wave equations can now be solved by a procedure analogous to the TTM [41], where we start with the transmitted field deep inside fused silica $(z>0)$ in the form $\sim e^{i k_{x} x+i k_{z, 2} z}$, with $k_{x}=(\omega / c) \sin \theta$ and $k_{z, 2}=(\omega / c)\left(\varepsilon_{\mathrm{SiO}_{2}}-\right.$ $\left.\sin ^{2} \theta\right)^{1 / 2}$ (this is the solution in the homogeneous material for $z \gg L$ where the permittivity is $\varepsilon_{\mathrm{SiO}_{2}}$ ) and integrate backwards to $z=0$. Next, the fields in the solid must be matched to the fields outside. For $p$-polarized light, the boundary conditions read

$$
\begin{aligned}
\lim _{z \rightarrow 0^{+}} B_{y}(z) & =\lim _{z \rightarrow 0^{-}} B_{y}(z), \\
\lim _{z \rightarrow 0^{+}} \frac{1}{\varepsilon_{x x}(z)} B_{y}^{\prime}(z) & =\lim _{z \rightarrow 0^{-}} \frac{1}{\varepsilon_{x x}(z)} B_{y}^{\prime}(z) .
\end{aligned}
$$

This provides the proper initial conditions for the field and its derivative in the second domain (air in this case). The conditions are equivalent for the $s$-polarized wave except that $E_{y}^{\prime}$ is continuous.

The field in air is represented by an incident and reflected plane wave of the form $F \sim e^{i k_{z, 1} z^{*}}+r e^{-i k_{z, 1} z^{*}}$, where $z^{*} \geqslant 0$. Here, $r$ is the complex reflection coefficient of interest, which we can now obtain as follows:

$$
r_{p}=\frac{i k_{z, 1} B_{y}^{*}-B_{y}^{\prime *}}{i k_{z, 1} B_{y}^{*}+B_{y}^{\prime *}}, \quad r_{s}=\frac{i k_{z, 1} E_{y}^{*}-E_{y}^{\prime *}}{i k_{z, 1} E_{y}^{*}+E_{y}^{\prime *}} .
$$

The starred quantities are the (numerically obtained) fields evaluated at $z^{*}$-for an abrupt air-solid interface as assumed in the main text, $z^{*}=0$. The reflectances are just $R_{p}=\left|r_{p}\right|^{2}$ and $R_{s}=\left|r_{s}\right|^{2}$.

The differential equations were solved using the DIFFERENTIALEQUATIONS.JL package [42] using nonstiff solvers. We checked in the isotropic case $\left(\varepsilon_{x x}=\varepsilon_{y y}=\varepsilon_{z z}\right)$ that this approach, solving the Helmholtz equations, gives the same reflectances as using the TMM (with the TMMPY package [43]) where the domain is sliced into many thin layers, each having a fixed permittivity.

The absorption coefficients (solid lines) in Fig. 7 were calculated from the time-averaged Poynting vector $\mathbf{S}=\operatorname{Re}(\mathbf{E} \times$ $\left.\mathbf{B}^{*}\right) / 2 \mu_{0}$ as $\boldsymbol{\nabla} \cdot \mathbf{S} /|\mathbf{S}|$.

\section{APPENDIX E: PARAMETERS IN THE ANISOTROPIC MODEL}

The optimized parameters in the anisotropic optical model are shown in Fig. 10 at different wavelengths. The observation that the parameters, which express the basic physical properties of the Drude plasma, $n_{0}$ and $\Gamma$, seem to be independent of probe wavelength gives credibility to the model. The delay for the onset of the anisotropic part of the permittivity $t_{0}$ is fairly small and appears to exhibit no specific trend on probe wavelength. The average values in Table I of the main text were obtained from the data shown in Fig. 10.

\section{APPENDIX F: BAYESIAN ANALYSIS OF REFLECTANCE DATA}

The steps outlined in Appendix A fully describe the dataanalysis procedure: the two crucial steps are the estimation of $\mathbf{x}^{(k)}(k=p, s)$ and the proper overlapping of the $p$ - and $s$-polarized sections on the camera. To carry out this procedure automatically and consistently, we apply Bayesian probability theory. The workflow is sketched in Fig. 11 using the methods described in this Appendix. We assume the reader is familiar 

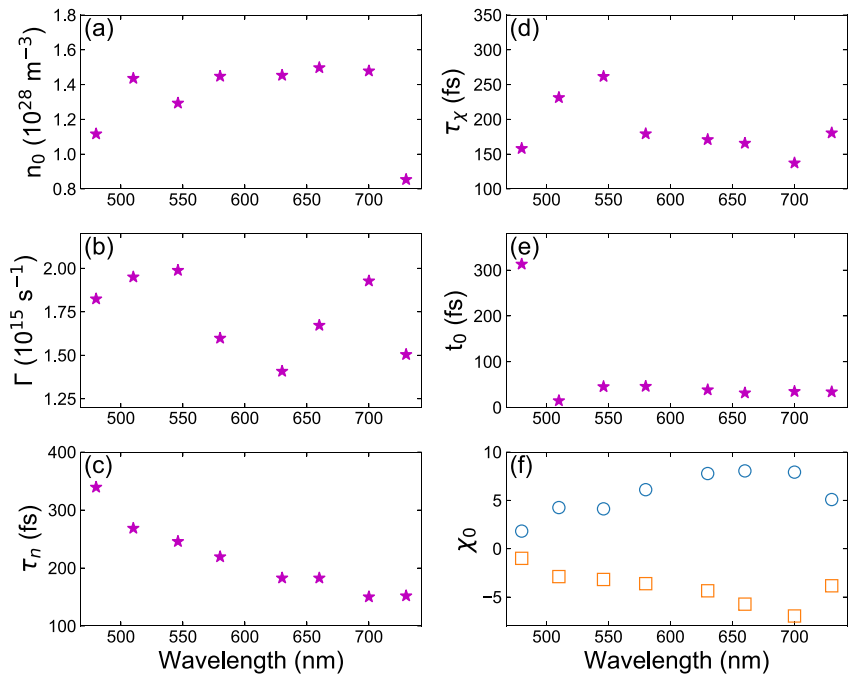

FIG. 10. (a)-(f) Parameters $n_{0}, \Gamma, \tau_{n}, \tau_{\chi}, t_{0}$, and $\chi_{0}$ in the anisotropic permittivity model defined in Eqs. (4)-(5), respectively. The blue circles and orange squares in (f) represent, respectively, the real and imaginary part of $\chi_{0}$.

with the basics of Bayesian probability theory (see, e.g., Ref. [44] for an introduction).

Equation (A1) is not an exact identity but subject to errors (experimental uncertainties), which we account for by adding an error term $\epsilon$ (suppressing positional $i$ indices):

$$
d=a(r+x)+\epsilon .
$$

We assume that the images have been normalized such that $0 \leqslant d \leqslant 1$. The errors result primarily from shot noise $(\epsilon \propto$ $\sqrt{d})$ since we are concerned with relatively large changes in reflectance due to the pump excitation. Consequently, the Poisson-distributed errors can be approximated by a Gaussian

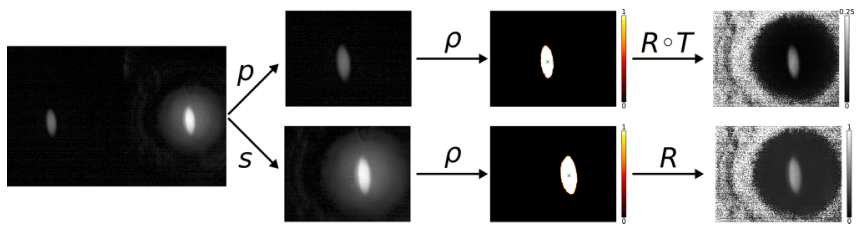

FIG. 11. Workflow (left to right) for obtaining $p$ and $s$ reflectances of fused silica when the reference $p$ reflectance is close zero. The first step is to split the image into the $p$ - and $s$-polarized sections. Then a map $\rho$ [Eq. (F18)] is applied separately to each section in order to "threshold" the pump-affected parts of the image ( 1 is associated with pump-induced signal, 0 is associated with the background). The center of the pump beam is found by computing the center of mass of the thresholded images (marked by green crosses). The $p$-polarized image is then translated $(T)$ to overlap the pump spot onto the $s$-polarized image. Finally, the reflectances of the $s$ and $p$ images are computed from Eqs. (A2) and (A3), respectively. Note that the regions with low probe signal produce an extreme variance in the reflectance (because of division by zero), but that is of no concern here since it is well outside the pump spot. probability distribution with standard error $\sigma$,

$$
P(\epsilon \mid \sigma I)=\frac{1}{\sqrt{2 \pi} \sigma} \mathrm{e}^{-\epsilon^{2} / 2 \sigma^{2}} .
$$

In our case, $\sigma=\sqrt{d / 2^{10}}$ (the factor $2^{10}$ originates from the normalization of the data and the bit depth of the camera).

Inspired by [44], we adopt a mixture-model approach, where a given pixel is classified according to whether it is outside $(T)$ or inside $(F)$ the pump spot. In the first case $(T)$, we know that $x \equiv 0$ and therefore $d=a r+\epsilon$. In the second case $(F)$, Eq. (F1) holds, and $x$ could be anything in the range from $-r$ to $a^{-1}-r$. We do not immediately know which of the two possibilities is more likely but suppose that a fraction $\beta$ of the image is not filled by the pump. Randomly drawn pixels from the image thus have a chance $\beta$ of containing no signal $(x=0)$. Therefore, the likelihood of observing datum $d$, given $r, a$, and $\beta$, is

$$
P(d \mid r a \beta I)=\beta P(d \mid r a T I)+(1-\beta) P(d \mid r a F I) .
$$

The likelihood when $T$ is true is, by virtue of Eqs. (F1) and (F2) with $x=0$, the familiar Gaussian likelihood:

$$
P(d \mid r a T I)=\frac{1}{\sqrt{2 \pi} \sigma} \mathrm{e}^{-(d-a r)^{2} / 2 \sigma^{2}} .
$$

When $F$ is true, the value of $x$ could, in principle, be anything in the range noted above. Therefore, we assign a uniform probability over $x$ :

$$
P(x \mid \operatorname{raFI})= \begin{cases}a^{-1} & \text { if }-r \leqslant x \leqslant a^{-1}-r, \\ 0 & \text { otherwise. }\end{cases}
$$

The likelihood for $d$ is in this case, without knowing $x$,

$$
\begin{aligned}
P(d \mid r a F I) & =\int d x P(x d \mid r a F I) \\
& =\int d x P(x \mid \operatorname{raFI}) P(d \mid \operatorname{rax} F I) \\
& =\frac{1}{2 a^{2}} \operatorname{erf}\left(\frac{d}{\sqrt{2} \sigma}\right)+\frac{1}{2 a^{2}} \operatorname{erf}\left(\frac{1-d}{\sqrt{2} \sigma}\right) .
\end{aligned}
$$

This specifies the mixture-model likelihood in Eq. (F3) for a single datum.

Suppose that $N$ samples have been chosen at random from the image: enumerate them $i=1,2, \ldots, N$. The full likelihood of $\mathbf{d}=\left\{d_{i}\right\}$ is just a product over the likelihoods of single data points:

$$
\begin{aligned}
P(\mathbf{d} \mid \mathbf{r} a \beta I)= & \prod_{i=1}^{N}\left\{\beta P\left(d_{i} \mid r_{i} a T I\right)\right. \\
& \left.+(1-\beta) P\left(d_{i} \mid r_{i} a F I\right)\right\} .
\end{aligned}
$$

From this likelihood, the posterior probability distribution for $a$ and $\beta$ is obtained through Bayes's theorem:

$$
P(a \beta \mid \mathbf{d r} I) \propto P(a \mid I) P(\beta \mid I) P(\mathbf{d} \mid \mathbf{r} a \beta I) .
$$

Prior distributions for $a$ and $\beta$ must be specified. For $a$ we choose a normal distribution centered around 1 with a width of 0.3 (the energy fluctuations of the probe are normally only about $10 \%$ ). $\beta$ is assigned a lopsided beta distribution, which restricts its values to the unit interval 
with more weight on large $\beta$ values (the pump normally fills about $5 \%-10 \%$ of the image d, suggesting that $\beta$ should be somewhere between 0.95 and 0.9 ). If sufficiently many samples are drawn from the images (typically, we draw $N=$ 10000 samples), the likelihood completely overrules the prior information. Therefore, the choice of the priors makes essentially no difference as long as they are consistent with the data.

The most probable values of $a$ and $\beta$, named $\hat{a}$ and $\hat{\beta}$, respectively, are found by maximizing either Eq. (F10) (maximum a posteriori estimation) or Eq. (F9) (maximum likelihood); if $N$ is large, the difference is immaterial.

To infer the reflectance, we need the posterior probability distribution for a particular pump-induced signal change $x_{j}$ :

$$
P\left(x_{j} \mid \mathbf{d r} I\right)=\int \operatorname{dad} \beta P(a \beta \mid \mathbf{d r} I) P\left(x_{j} \mid \mathbf{d r} a \beta I\right) .
$$

In practice, the probability distributions for $a$ and $\beta$ are so narrow that the uncertainties they produce in the uncertainty analysis of the reflectances are negligible compared to the shot noise error $\sigma$. We will therefore make the simplifying approximation that $P(a \beta \mid \mathbf{d r} I) \approx \delta(a-\hat{a}) \delta(\beta-\hat{\beta})$. In this approximation, Eq. (F11) reduces to

$$
\begin{aligned}
P\left(x_{j} \mid \mathbf{d r} I\right) \propto & \left\{\hat{\beta} \delta\left(x_{j}\right) P\left(d_{j} \mid r_{j} \hat{a} T I\right)\right. \\
& \left.+(1-\hat{\beta}) P\left(x_{j} \mid r_{j} \hat{a} F I\right) P\left(d_{j} \mid r_{j} x_{j} \hat{a} F I\right)\right\} .
\end{aligned}
$$

The probability distribution of $x_{j}$ is (within the approximations) a simple average of the product of the likelihoods and priors associated with the two possibilities $T$ (sample $j$ outside the pump spot) and $F$ (inside pump spot), weighted according to $\hat{\beta}$ and $1-\hat{\beta}$.
Suppose that $F$ is true ( $x_{j}$ is nonzero) and neglect the first term in Eq. (F12). Reflectance changes, which are proportional to $x_{j}$, are everywhere (e.g., in Fig. 5) represented in terms of the average value and standard deviation of $x_{j}$ : $\Delta R_{j} \propto \bar{x}_{j} \pm \sqrt{\overline{x_{j}^{2}}-\bar{x}_{j}^{2}}$. The required moment integrals can be carried out analytically:

$$
\begin{aligned}
\overline{x^{n}} & \equiv \int d x x^{n} P(x \mid \mathbf{d r} F I) \\
& =\frac{1}{Z_{n}} \sum_{k=0}^{n}\left(\begin{array}{l}
n \\
k
\end{array}\right)(-1)^{k}\left(\frac{d-a r}{\sqrt{2} \sigma}\right)^{n-k}\left(\mathcal{I}_{k}-\mathcal{J}_{k}\right),
\end{aligned}
$$

where

$$
\begin{gathered}
Z_{n}=\sqrt{\pi}\left(\frac{a}{\sqrt{2} \sigma}\right)^{n}\left[\operatorname{erf}\left(\frac{d}{\sqrt{2} \sigma}\right)+\operatorname{erf}\left(\frac{1-d}{\sqrt{2} \sigma}\right)\right], \\
\mathcal{I}_{k}=\left[1+(-1)^{k}\right] \Gamma\left(m_{k}\right), \\
\mathcal{J}_{k}=(-1)^{k} \Gamma\left(m_{k} ;(1-d)^{2} / 2 \sigma^{2}\right) \\
+\Gamma\left(m_{k} ; d^{2} / 2 \sigma^{2}\right) .
\end{gathered}
$$

$\Gamma$ is the (incomplete) gamma function, and $m_{k}=(k+1) / 2$.

Finally, it is possible to compute the probability $\rho$ that a particular sample is within the beam spot. Skipping the details (see [44]) and using the previous approximations,

$$
\rho=\frac{(1-\hat{\beta}) P(d \mid r \hat{a} F I)}{(1-\hat{\beta}) P(d \mid r \hat{a} F I)+\hat{\beta} P(d \mid r \hat{a} T I)} .
$$

This is just a weighted ratio average of the likelihoods for $F$ and $T$, which certainly makes a lot of sense. Equation (F18) is used to threshold the pump spot in the $p$ - and $s$-polarized sections of the images to properly overlap them (see Fig. 11).
[1] L. V. Keldysh, Ionization in the field of a strong electromagnetic wave, Sov. Phys. JETP 20, 1307 (1965).

[2] B. Rethfeld, Free-electron generation in laser-irradiated dielectrics, Phys. Rev. B 73, 035101 (2006).

[3] P. Balling and J. Schou, Femtosecond-laser ablation dynamics of dielectrics: Basics and applications for thin films, Rep. Prog. Phys. 76, 036502 (2013).

[4] B. Rethfeld, D. S. Ivanov, M. E. Garcia, and S. I. Anisimov, Modelling ultrafast laser ablation, J. Phys. D 50, 193001 (2017).

[5] C. Thaury, F. Quéré, J.-P. Geindre, A. Levy, T. Ceccotti, P. Monot, M. Bougeard, F. Réau, P. d'Oliveira, P. Audebert, R. Marjoribanks, and P. Martin, Plasma mirrors for ultrahighintensity optics, Nat. Phys. 3, 424 (2007).

[6] K. Sokolowski-Tinten, J. Bialkowski, and D. von der Linde, Ultrafast laser-induced order-disorder transitions in semiconductors, Phys. Rev. B 51, 14186 (1995).

[7] L. Huang, J. P. Callan, E. N. Glezer, and E. Mazur, GaAs under Intense Ultrafast Excitation: Response of the Dielectric Function, Phys. Rev. Lett. 80, 185 (1998).

[8] F. Quéré, S. Guizard, P. Martin, G. Petite, O. Gobert, P. Meynadier, and M. Perdrix, Ultrafast carrier dynamics in laser- excited materials: Subpicosecond optical studies, Appl. Phys. B 68, 459 (1999).

[9] C. Quoix, G. Hamoniaux, A. Antonetti, J.-C. Gauthier, J.-P. Geindre, and P. Audebert, Ultrafast plasma studies by phase and amplitude measurements with femtosecond spectral interferometry, J. Quant. Spectrosc. Radiat. Transfer 65, 455 (2000).

[10] K. Sokolowski-Tinten and D. von der Linde, Generation of dense electron-hole plasmas in silicon, Phys. Rev. B 61, 2643 (2000).

[11] D. Puerto, W. Gawelda, J. Siegel, J. Bonse, G. Bachelier, and J. Solis, Transient reflectivity and transmission changes during plasma formation and ablation in fused silica induced by femtosecond laser pulses, Appl. Phys. A 92, 803 (2008).

[12] M. Garcia-Lechuga, J. Siegel, J. Hernandez-Rueda, and J. Solis, Imaging the ultrafast Kerr effect, free carrier generation, relaxation and ablation dynamics of lithium niobate irradiated with femtosecond laser pulses, J. Appl. Phys. 116, 113502 (2014).

[13] F. Meng, M. D. Thomson, B. E. Sernelius, M. Jörger, and H. G. Roskos, Ultrafast dynamic conductivity and scattering rate saturation of photoexcited charge carriers in silicon investigated with a midinfrared continuum probe, Phys. Rev. B 91, 075201 (2015). 
[14] L. Haahr-Lillevang, K. Wædegaard, D. B. Sandkamm, A. Mouskeftaras, S. Guizard, and P. Balling, Short-pulse laser excitation of quartz: Experiments and modeling of transient optical properties and ablation, Appl. Phys. A 120, 1221 (2015).

[15] T. Kumada, T. Otobe, M. Nishikino, N. Hasegawa, and T. Hayashi, Dynamics of spallation during femtosecond laser ablation studied by time-resolved reflectivity with double pump pulses, Appl. Phys. Lett. 108, 011102 (2016).

[16] M. Garcia-Lechuga, L. Haahr-Lillevang, J. Siegel, P. Balling, S. Guizard, and J. Solis, Simultaneous time-space resolved reflectivity and interferometric measurements of dielectrics excited with femtosecond laser pulses, Phys. Rev. B 95, 214114 (2017).

[17] T. Winkler, L. Haahr-Lillevang, C. Sarpe, B. Zielinski, N. Götte, A. Senftleben, P. Balling, and T. Baumert, Laser amplification in excited dielectrics, Nat. Phys. 14, 74 (2017).

[18] T. Winkler, P. Balling, B. Zielinski, C. Sarpe, N. Jelzow, R. Ciobotea, A. Senftleben, and T. Baumert, Unveiling nonlinear regimes of light amplification in fused silica with femtosecond imaging spectroscopy, Phys. Rev. Res. 2, 023341 (2020).

[19] C. Fourment, B. Chimier, F. Deneuville, D. Descamps, F. Dorchies, G. Duchateau, M.-C. Nadeau, and S. Petit, Ultrafast changes in optical properties of $\mathrm{SiO}_{2}$ excited by femtosecond laser at the damage threshold and above, Phys. Rev. B 98, 155110 (2018).

[20] J. Winter, S. Rapp, M. Spellauge, C. Eulenkamp, M. Schmidt, and H. P. Huber, Ultrafast pump-probe ellipsometry and microscopy reveal the surface dynamics of femtosecond laser ablation of aluminium and stainless steel, Appl. Surf. Sci. 511, 145514 (2020).

[21] H. Tompkins and E. A. Irene, Handbook of Ellipsometry (Springer-Verlag, Heidelberg, 2005).

[22] I. H. Malitson, Interspecimen comparison of the refractive index of fused silica, J. Opt. Soc. Am. 55, 1205 (1965).

[23] A. Rämer, L. Haahr-Lillevang, B. Rethfeld, and P. Balling, Modeling the transient optical parameters in laser-excited band gap materials, Opt. Eng. 56, 011015 (2016).

[24] M. H. Javani and M. I. Stockman, Real and Imaginary Properties of Epsilon-Near-Zero Materials, Phys. Rev. Lett. 117, 107404 (2016).

[25] K. Kim, Resonant absorption of electromagnetic waves in transition anisotropic media, Opt. Express 25, 30162 (2017).

[26] A. Ciattoni, A. Marini, C. Rizza, M. Scalora, and F. Biancalana, Polariton excitation in epsilon-near-zero slabs: Transient trapping of slow light, Phys. Rev. A 87, 053853 (2013).

[27] S. Campione, I. Kim, D. de Ceglia, G. A. Keeler, and T. S. Luk, Experimental verification of epsilon-near-zero plasmon polariton modes in degenerately doped semiconductor nanolayers, Opt. Express 24, 18782 (2016).

[28] N. Brouwer and B. Rethfeld, Excitation and relaxation dynamics in dielectrics irradiated by an intense ultrashort laser pulse, J. Opt. Soc. Am. B 31, C28 (2014).
[29] B. Rethfeld, A. Rämer, N. Brouwer, N. Medvedev, and O. Osmani, Electron dynamics and energy dissipation in highly excited dielectrics, Nucl. Instrum. Methods Phys. Res. 327, 78 (2014).

[30] W. Kruer, The Physics of Laser Plasma Interactions (AddisonWesley, Redwood City, CA, 1988).

[31] R. Loudon, The propagation of electromagnetic energy through an absorbing dielectric, J. Phys. A 3, 233 (1970).

[32] J. Lekner, Reflection and refraction by uniaxial crystals, J. Phys.: Condens. Matter 3, 6121 (1991).

[33] P. Balling, Laser coupling and relaxation of the absorbed energy: Metals, semiconductors and dielectrics, in Handbook of Laser Micro- and Nano-engineering, edited by K. Sugioka (Springer, Cham, 2020), pp. 1-57.

[34] S. A. Sato, K. Yabana, Y. Shinohara, T. Otobe, and G. F. Bertsch, Numerical pump-probe experiments of laser-excited silicon in nonequilibrium phase, Phys. Rev. B 89, 064304 (2014).

[35] X. Zhang, F. Wang, L. Jiang, and Y. Yao, Manipulation of the dielectric properties of diamond by an ultrashort laser pulse, Phys. Rev. B 95, 184301 (2017).

[36] M. Qasim, M. S. Wismer, M. Agarwal, and V. S. Yakovlev, Ensemble properties of charge carriers injected by an ultrashort laser pulse, Phys. Rev. B 98, 214304 (2018).

[37] J. Sjakste, K. Tanimura, G. Barbarino, L. Perfetti, and N. Vast, Hot electron relaxation dynamics in semiconductors: Assessing the strength of the electron-phonon coupling from the theoretical and experimental viewpoints, J. Phys.: Condens. Matter 30, 353001 (2018).

[38] D. Grojo, M. Gertsvolf, S. Lei, T. Barillot, D. M. Rayner, and P. B. Corkum, Exciton-seeded multiphoton ionization in bulk $\mathrm{SiO}_{2}$, Phys. Rev. B 81, 212301 (2010).

[39] R. Stoian, A. Rosenfeld, D. Ashkenasi, I. V. Hertel, N. M. Bulgakova, and E. E. B. Campbell, Surface Charging and Impulsive Ion Ejection During Ultrashort Pulsed Laser Ablation, Phys. Rev. Lett. 88, 097603 (2002).

[40] N. M. Bulgakova, R. Stoian, A. Rosenfeld, I. V. Hertel, and E. E. B. Campbell, Electronic transport and consequences for material removal in ultrafast pulsed laser ablation of materials, Phys. Rev. B 69, 054102 (2004).

[41] S. J. Byrnes, Multilayer optical calculations, arXiv:1603.02720.

[42] C. Rackauckas and Q. Nie, DifferentialEquations.j1 - A performant and feature-rich ecosystem for solving differential equations in Julia, J. Open Res. Software 5, 15 (2017).

[43] E. H. Eriksen, TMMPY, 2018, https://zenodo.org/record/ 1344878.

[44] W. Von der Linden, V. Dose, and U. Von Toussaint, Bayesian Probability Theory: Applications in the Physical Sciences (Cambridge University Press, Cambridge, 2014). 